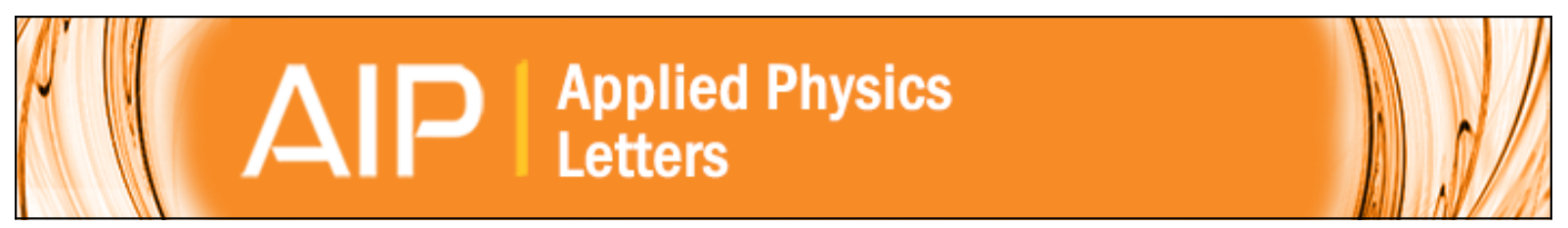

\title{
Terahertz beam focusing based on plasmonic waveguide scattering
}

Yasuaki Monnai, Kristian Altmann, Christian Jansen, Martin Koch, Hartmut Hillmer, and Hiroyuki Shinoda

Citation: Applied Physics Letters 101, 151116 (2012); doi: 10.1063/1.4759042

View online: http://dx.doi.org/10.1063/1.4759042

View Table of Contents: http://scitation.aip.org/content/aip/journal/apl/101/15?ver=pdfcov

Published by the AIP Publishing

\section{Advertisement:}

\section{AlP Re-register for Table of Content Alerts}




\title{
Terahertz beam focusing based on plasmonic waveguide scattering
}

\author{
Yasuaki Monnai, ${ }^{1, a)}$ Kristian Altmann, ${ }^{2}$ Christian Jansen, ${ }^{2}$ Martin Koch, ${ }^{2}$ Hartmut Hillmer, ${ }^{3}$ \\ and Hiroyuki Shinoda ${ }^{1}$ \\ ${ }^{1}$ Department of Information Physics and Computing, The University of Tokyo, 7-3-1 Hongo, Bunkyo-ku, \\ Tokyo 113-8656, Japan \\ ${ }^{2}$ Department of Physics, Philipps University of Marburg, Renthof 5, 35032 Marburg, Germany \\ ${ }^{3}$ Institute of Nanostructure Technologies and Analytics, University of Kassel, \\ Heinrich-Plett-Str. 40, 34132 Kassel, Germany
}

(Received 25 July 2012; accepted 1 October 2012; published online 11 October 2012)

\begin{abstract}
We demonstrate free-space focusing of terahertz $(\mathrm{THz})$ radiation by scattering plasmonic surface-waves into the air. We use a grating of shallow holes which contains non-equidistant defects which act as scattering centers. The scattering occurs with defined phase delays such that the waves emitted in free-space interfere constructively to form a focus above the waveguide surface. In contrast to conventional lenses, this structure does not require any free-space on its backside and has great potential for integrated THz optics. (C) 2012 American Institute of Physics. [http://dx.doi.org/10.1063/1.4759042]
\end{abstract}

Lenses are fundamental components in terahertz $(\mathrm{THz})$ systems. Compared to their optical counterparts, THz lenses are bulky due to the relatively long wavelengths. ${ }^{1,2}$ Recently, several attempts have been reported to fabricate thin $\mathrm{THz}$ lenses based on Fresnel zone plates or using metamaterials. ${ }^{3-6}$ Even though the thickness of each lens could thus be reduced, the entire $\mathrm{THz}$ system remains bulky as long as the system relies on free-space propagation paths between the lenses.

In this letter, we demonstrate a planar $\mathrm{THz}$ lens based on the scattering of surface-waves from a plasmonic waveguide with a chirped-grating structure. Since the radiation aperture is excited by guided waves, no additional free-space is required on the backside of the structure. So far, in the context of integrated optics, chirped-gratings grooved on dielectric waveguides have been shown to form an optical focus based on constructive interference of the scattered light in the air. ${ }^{7}$ Here, we point out that metallic grating couplers hold great potential for integrated $\mathrm{THz}$ devices. They not only allow for low-loss surface-wave transmission ${ }^{8-11}$ but also for a variety of plasmonic functional components. ${ }^{12-14}$ The use of metal also assures that the radiation appears only above the structure and prevents so-called substrate modes as in dielectric structures. ${ }^{15}$ All of these features are favorable for developing integrated $\mathrm{THz}$ systems interfaced with the surrounding spaces.

Recently, the authors have numerically presented that spatially perturbed metal corrugations generate focused $\mathrm{THz}$ radiation. ${ }^{16}$ There, the corrugation was modeled as a genuine 1D periodic structure uniform in the lateral direction. For practical devices, it is important to incorporate a mechanism that prevents two-dimensional lateral spreading of the surface-wavefront. The structure demonstrated in this letter is based on a "blind" hole array, ${ }^{17}$ which supports onedimensionally confined surface-waves propagating along hole apertures grooved on metal surfaces. We implemented chirped gratings on such a waveguide by filling a series of the holes as shown in Fig. 1(a). The partially flat regions

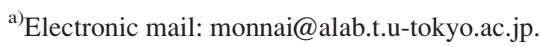

disturb the surface-waves and scatter them into the air with defined phase delays. To illustrate the scattering principle, we compare two simulations calculated with CST MWStudio in Figs. 1(c) and 1(d). In Fig. 1(c), there are no holes filled, i.e., the hole array is perfectly periodic working as a normal waveguide. In Fig. 1(d), there is one hole filled, which scatters the incoming surface-wave into the air serving as a point source. Focusing is achieved by designing the grating pattern in a way that each scattered wave contributes to constructive interference at a single point in the air. Note, that the phase velocity of the surface-wave is slower than that of free-space. The focus should be line-shaped spreading in the $y$-direction as in the case of cylindrical lenses since the structure has no mechanisms to focus the radiation in that direction (Fig. 2).

The structure was fabricated out of stainless steel (SUS304). The blind holes were processed in two steps.
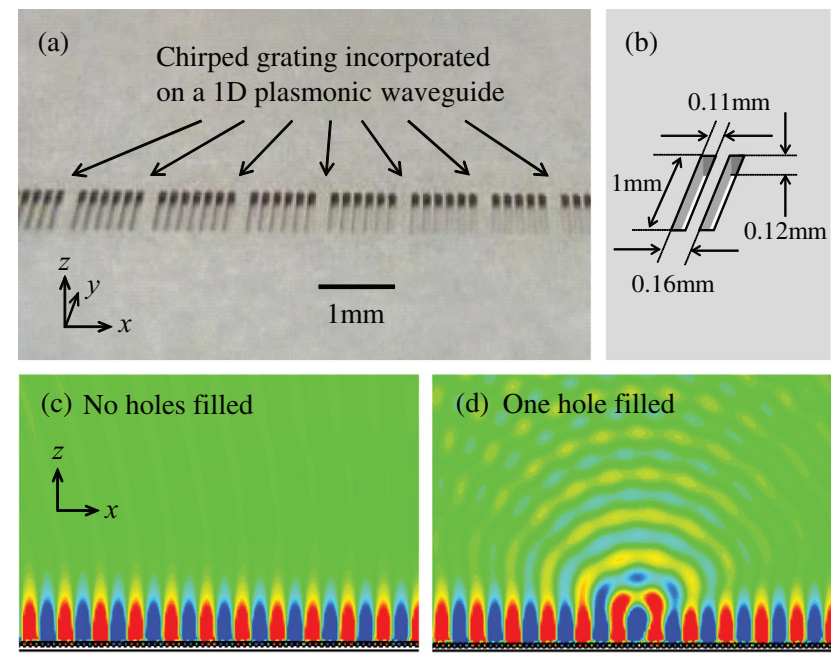

FIG. 1. (a) Photograph of the developed structure. A series of the holes are filled to form a chirped grating pattern. (b) Definition of the dimensions. The waveguide is defined by rectangular shaped holes of $0.12 \mathrm{~mm}$ deep aligned in $0.16 \mathrm{~mm}$ period. (c) Simulation with no holes filled. (d) Simulation with one hole filled. 


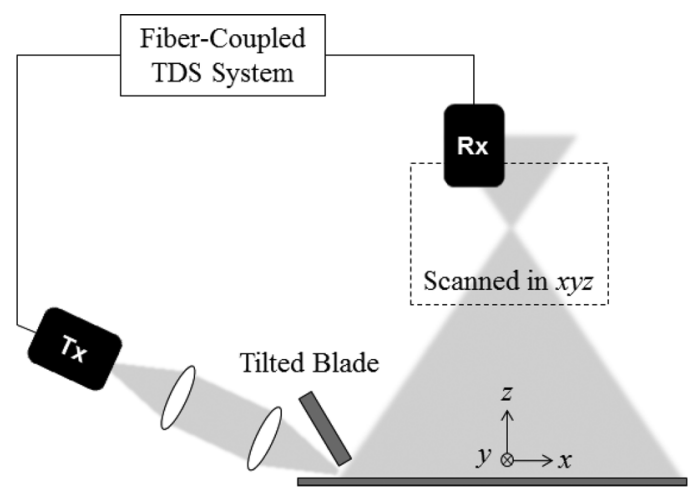

1D Plasmonic Waveguide with Chirped Grating

FIG. 2. Experimental setup. The receiver moves in $x y z$ to map the field above the waveguide. The fields are kept in TM-polarization throughout the measurement (the magnetic field has only $y$-components).

Through-holes were first created with a standard photoetching process and then the bottom was covered with a continuous flat plate using thermo-compression bonding. Stainless steel possesses less than one-tenth of the conductivity of aluminum. Yet, we used that material for its capability of etching very fine structures. It is yet possible to increase the surface conductivity by coating the surface with another metal like aluminum. ${ }^{17}$ The hole dimensions are summarized in Fig. 1(b). The holes have a nearly rectangular shape and a depth of $0.12 \mathrm{~mm}$. The hole array has a period of $0.16 \mathrm{~mm}$ and the total length of $40 \mathrm{~mm}$. The anti-resonant (AR) frequency, ${ }^{17}$ above which the surface-wave propagation is prohibited was estimated to be around $420 \mathrm{GHz}$. Given the dispersion curve by CST, the chirped grating pattern was designed so that a focus is formed $30 \mathrm{~mm}$ above the waveguide at $300 \mathrm{GHz}$. Due to the dispersive nature of the structure, the focus moves as the frequency changes forming a trace as predicted in Fig. 3(a) (solid curve).

The focus generated by the fabricated device is experimentally characterized using a fiber-coupled THz-time domain

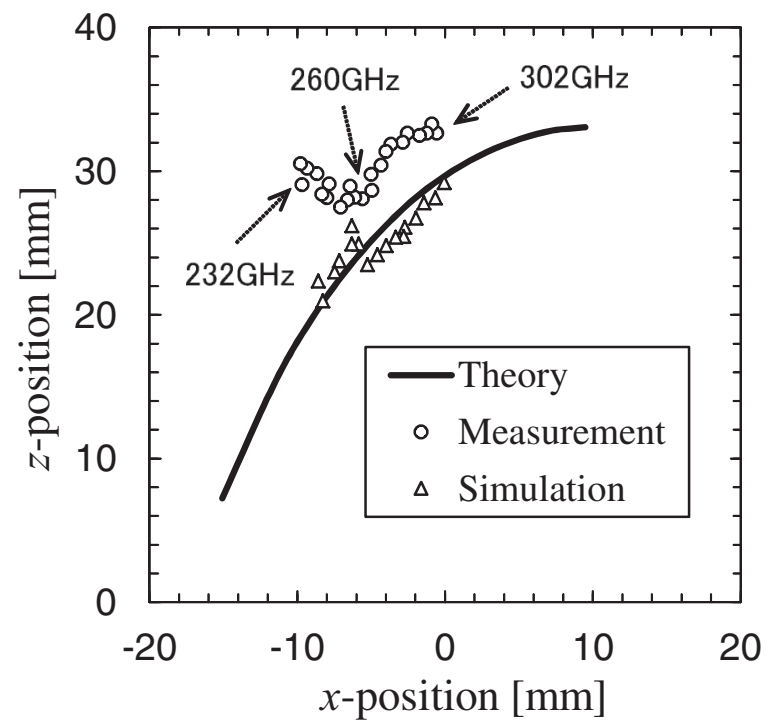

FIG. 3. Frequency-dependent trace of the focus. Solid curve represents a theoretical curve while circles and triangles express the measurement (from $232 \mathrm{GHz}$ to $302 \mathrm{GHz}$ at about $3 \mathrm{GHz}$ intervals) and simulation $(230 \mathrm{GHz}$ to $300 \mathrm{GHz}$ at about $5 \mathrm{GHz}$ intervals), respectively.

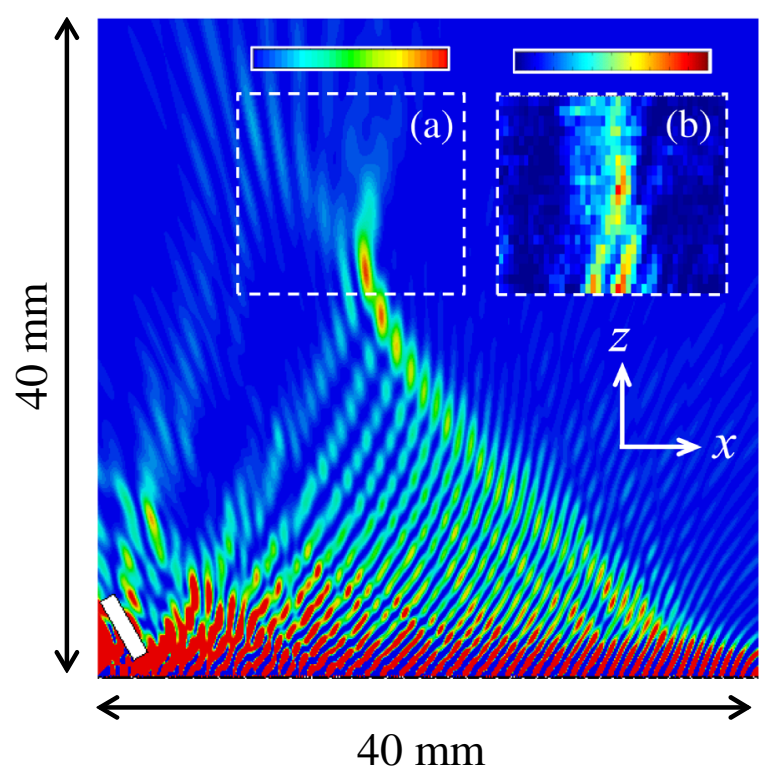

FIG. 4. Focused radiation observed at $270 \mathrm{GHz}$ (power distribution). (a) Simulation and (b) measurement. The measured area is indicated by the dashed squares.

spectroscopy (TDS) system (Fig. 2). TM-polarized THz pulses are coupled onto the waveguide through a narrow slit between the waveguide surface and a metal blade separated by $1 \mathrm{~mm}$. The blade is tilted by $30^{\circ}$ from the $z$-direction so that scattered waves reflected by the blade do not disturb the focused radiation. The field distribution above the waveguide is mapped by scanning the receiver. Since the surface-wave is TM-polarized, the focus is also TM-polarized. Figure 4 compares the field distribution between (a) simulation and (b) measurement at $270 \mathrm{GHz}$. They show good qualitative agreement and the focusing effect is clearly observed. The field pattern observed in parallel to the focal plane is shown in Fig. 5 confirming a clear line-shaped focus. During the measurement, we observed shorter lines compared to the simulation. This can be explained by the reduced sensitivity of the receiver for larger incident angles. The frequency-dependent trace of the focus from $232 \mathrm{GHz}$ to $302 \mathrm{GHz}$ is plotted in Fig. 3 (circles) along with

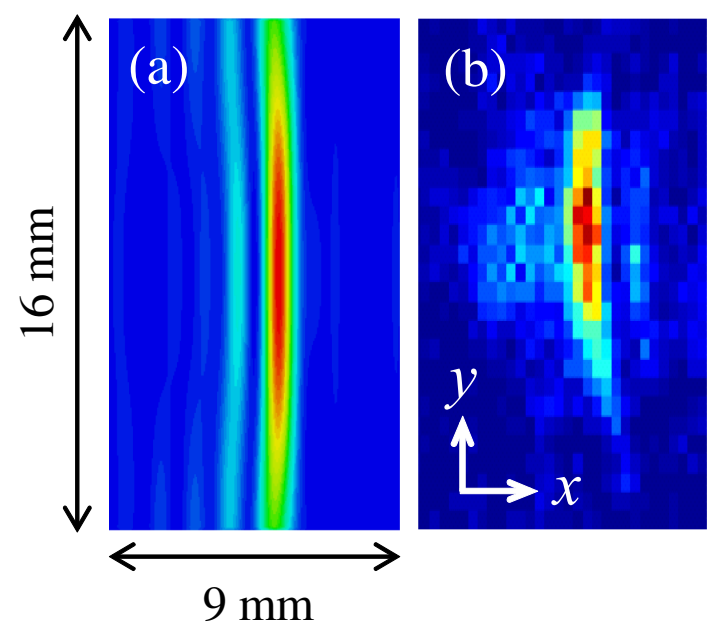

FIG. 5. Line-shaped focus observed at $270 \mathrm{GHz}$ in parallel to the focal plane (power distribution). (a) Simulation $(z=24.8 \mathrm{~mm})$ and (b) measurement $(z=28 \mathrm{~mm})$. The color bar is the same as in Fig. 4 . 


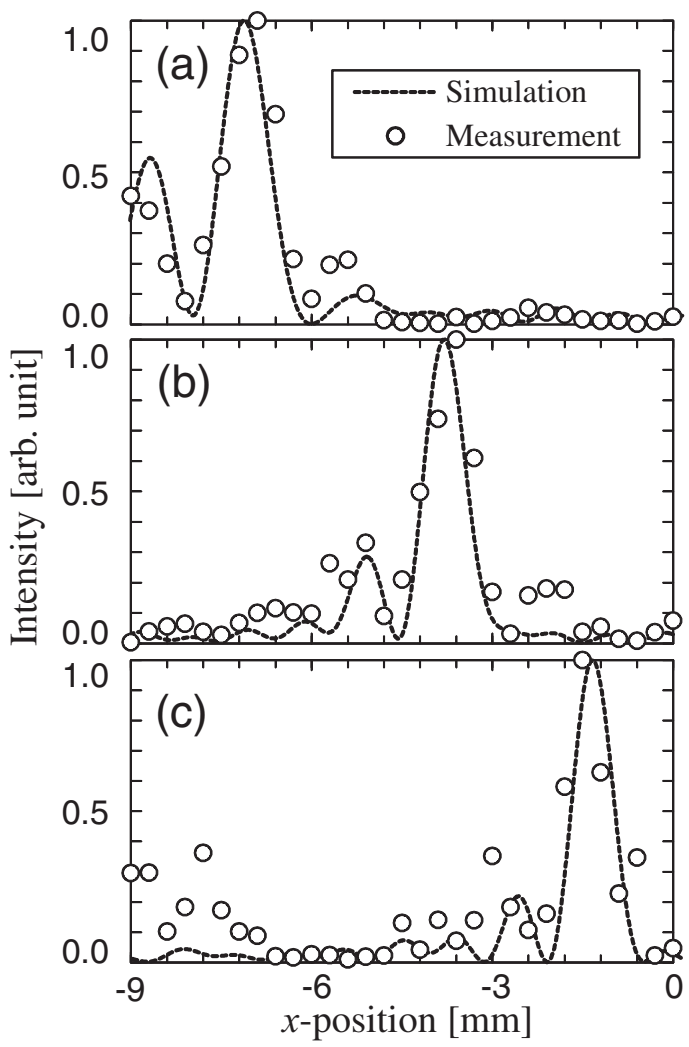

FIG. 6. Profiles of the focus at (a) $243 \mathrm{GHz}$, (b) $270 \mathrm{GHz}$, and (c) $290 \mathrm{GHz}$, respectively. The $z$-position of the profiles differs between the simulation and measurement as indicated in Fig. 3.

the simulation (triangles). The focus always moves in positive $x$-direction as the frequency increases. In the experiment, the focus appeared several millimeters higher than expected from the simulation. We attribute this to a silicon lens on the receiver that shifts the apparent focal position. The measurement and the simulation showed the same tendency as the theoretical curve above $260 \mathrm{GHz}$. Below that frequency, the behavior changed because of interference with unintentionally diffracted radiation emanating from the incoupling slit. The measurement above $300 \mathrm{GHz}$ almost reached the noise level and the focus was hardly observable. In order to increase the signal-to-noise ratio, it is important to reduce the conductive surface losses, which increase with frequency. The surface-waves also experience attenuation due to the radiation losses. This makes the contribution from the last scatterers weaker than the first ones, resulting in non-uniform wavefronts. This could be compensated by spatially modulating the shape of the grating. Focusing the radiation in the $y$-direction using twodimensionally aligned chirped-gratings could also contribute to improve the signal. Figure 6 shows the focal profiles at (a) $243 \mathrm{GHz}$, (b) $270 \mathrm{GHz}$, and (c) $290 \mathrm{GHz}$, respectively. The focal widths (FWHM) are (a) $0.89 \mathrm{~mm}(0.72 \lambda)$, (b) $0.77 \mathrm{~mm}$ $(0.69 \lambda)$, and (c) $0.74 \mathrm{~mm}(0.72 \lambda)$, respectively, and are almost diffraction limited. As mentioned earlier the focus position is frequency dependent. From the CST simulation, we roughly estimated the loss budget of the system at $285 \mathrm{GHz}$ as follows: $40 \%$ of the excited surface-wave was radiated into the air (16\% was focused within the FWHM and $24 \%$ was stray light), another $40 \%$ was dissipated by the stainless-steel (the conductivity of $1.4 \times 10^{6}[\mathrm{~S} / \mathrm{m}]^{18}$ ), and the remaining $20 \%$ was transmitted to the other end of the waveguide without being scattered or dissipated. In conclusion, we have demonstrated $\mathrm{THz}$ beam focusing based on plasmonic waveguide scattering. Although we used the structure as a transmitter in this letter, it could also be used as a receiving lens. Other beam shapes, i.e., diffraction patterns can also be tailored by designing the grating pattern based on the waveguide hologram concept. ${ }^{19}$ The blind hole array structure holds potential to be further engineered, for example, by filling the holes with active or tunable materials, which give prospects for future smart THz systems.

The research was partly supported by Grant-in-Aid for the JSPS Fellows (22-4238) and National Institute of Information and Communications Technology (NICT) 13701.

${ }^{1}$ B. Scherger, C. Jördens, and M. Koch, Opt. Express 19, 4528 (2011).

${ }^{2}$ B. Scherger, M. Scheller, C. Jansen, M. Koch, and K. Wiesauer, Appl. Opt. 50, 2256 (2011).

${ }^{3}$ S. Wang, T. Yuan, E. D. Walsby, R. J. Blaikie, S. M. Durbin, D. R. S. Cumming, J. Xu, and X. C. Zhang, Opt. Lett. 27, 1183 (2002).

${ }^{4}$ E. D. Walsby, J. Alton, C. Worrall, H. E. Beere, D. A. Ritchie, and D. R. S. Cumming, Opt. Lett. 32, 1141 (2007).

${ }^{5}$ J. Neu, B. Krolla, O. Paul, B. Reinhard, R. Beigang, and M. Rahm, Opt. Express 18, 27748 (2010).

${ }^{6}$ A. Siemion, A. Siemion, M. Makowski, M. Sypek, E. Hrault, F. Garet, and J. L. Coutaz, Opt. Lett. 36, 1960 (2011).

${ }^{7}$ A. Katzir, A. C. Livanos, and A. Yariv, Appl. Phys. Lett. 30, 225 (1977).

${ }^{8}$ K. Wang and D. M. Mittleman, Nature 432, 376 (2004).

${ }^{9}$ T. I. Jeon and D. Grischkowsky, Appl. Phys. Lett. 88, 061113 (2006).

${ }^{10}$ R. F. Oulton, V. J. Sorger, D. A. Genov, D. F. P. Pile, and X. Zhang, Nature Photon. 2, 496 (2008).

${ }^{11}$ L. Shen, X. Chen, and T. J. Yang, Opt. Express 16, 3326 (2008).

${ }^{12}$ D. Wu, N. Fang, C. Sun, X. Zhang, W. J. Padilla, D. N. Basov, D. R. Smith, and S. Schultz, Appl. Phys. Lett. 83, 201 (2003).

${ }^{13}$ B. Wang and G. P. Wang, Appl. Phys. Lett. 89, 133106 (2006).

${ }^{14}$ Q. Gan, Z. Fu, Y. J. Ding, and F. J. Bartoli, Phys. Rev. Lett. 100, 256803 (2008).

${ }^{15} \mathrm{H}$. Nishihara, M. Haruna, and T. Suhara, Optical Integrated Circuits (McGraw-Hill, 1989).

${ }^{16}$ Y. Monnai, H. Shinoda, and H. Hillmer, Appl. Phys. B 104, 913 (2011).

${ }^{17}$ G. Kumar, S. Pandey, A. Cui, and A. Nahata, New J. Phys. 13, 033024 (2011).

${ }^{18}$ Metals Handbook, Desk Edition, edited by J. R. Davis (ASM International, 1998).

${ }^{19}$ M. Li, J. Bengtsson, M. Hagberg, A. Larsson, and T. Suhara, IEEE J. Sel. Top. Quantum Electron. 2, 226 (1996). 